\title{
La déclaration de repentir dans la Grèce des années
} 1940

\section{Christina Alexopoulos}

\section{OpenEdition}

1 Journals

Édition électronique

URL : https://journals.openedition.org/ceb/773

DOI : $10.4000 /$ ceb.773

ISSN : 2261-4184

Éditeur

INALCO

Édition imprimée

Date de publication : 30 mars 2011

Pagination : 85-98

ISBN : 978-2-85831-189-7

ISSN : 0290-7402

\section{Référence électronique}

Christina Alexopoulos, "La déclaration de repentir dans la Grèce des années 1940 », Cahiers

balkaniques [En ligne], 38-39 | 2011, mis en ligne le 30 novembre 2011, consulté le 06 juillet 2021. URL : http://journals.openedition.org/ceb/773 ; DOI : https://doi.org/10.4000/ceb.773

Ce document a été généré automatiquement le 6 juillet 2021.

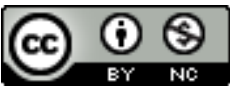

Cahiers balkaniques est mis à disposition selon les termes de la Licence Creative Commons Attribution - Pas d'Utilisation Commerciale 4.0 International. 


\title{
La déclaration de repentir dans la Grèce des années 1940
}

\author{
Christina Alexopoulos
}

\section{Introduction}

1 En grec moderne, l'expression "signer sa déclaration de repentir», désigne communément un mea-culpa public, humiliant et injustifié, un dénigrement systématique et total de ses actes et pensées, un déni pur et simple de son identité, ou encore mieux un aveu de défaite inconditionnelle, une résignation honteuse à la raison du plus fort. Or, il faut savoir qu'avant de se transformer en locution figée nettement péjorative, la déclaration de repentir, a existé comme document officiel attestant la conversion idéologique de son signataire.

2 Elle était obtenue généralement de force, souvent dans des espaces de réclusion à caractère concentrationnaire ou tout au moins coercitif (camps de déportation et/ou de rééducation, prisons, locaux de la Sûreté nationale, hôpitaux militaires, asiles), et servait aussi bien à montrer la victoire de l'idéologie dominante sur des valeurs jugées subversives, qu'à discréditer les signataires auprès de leurs anciens compagnons de lutte, en les contraignant au silence et au déshonneur.

3 Arme de propagande idéologique et expression de l'anticommunisme ambiant, la déclaration de repentir a été largement employée par les régimes grecs avant, pendant et après la guerre civile grecque en s'investissant de nouvelles fonctions au fil du temps. La déclaration de repentir trouve ses racines dans les pratiques de la Grèce des années vingt, voit son usage s'intensifier dans les années 1930, notamment sous la dictature de Métaxas, avant de se transformer en un enjeu majeur de la guerre civile grecque, considérée à juste titre comme le premier conflit armé de la guerre froide. Très présente dans la période qui a succédé à la guerre civile, la déclaration de repentir ne disparaît du paysage politique grec qu'à la chute de la junte des colonels, quand les partis communistes grecs sont légalisés. 
4 Associée à différents certificats de «bonnes convictions nationales ", la déclaration de repentir vient sceller la répartition des Grecs en bons et mauvais citoyens, séparant, pour reprendre la connotation religieuse du texte, le bon grain de l'ivraie. Très manichéenne dans sa conception, elle est révélatrice du climat politique de la période de la guerre civile. Elle éclaire la manière dont les différents régimes politiques grecs ont appréhendé l'idéologie communiste et en dit long sur les objectifs et les méthodes des vainqueurs de la guerre civile.

Dans les limites de la présente étude, nous tenterons de replacer la déclaration de repentir dans son contexte historique, puis d'étudier le discours et les soubassements idéologiques du texte de la déclaration. Enfin, nous essaierons de comprendre son fonctionnement, son impact psychologique sur la subjectivité des signataires et son incidence sur la vie politique et sociale du pays.

\section{Historique de la déclaration de repentir}

\section{La déclaration de repentir depuis sa création jusqu'aux années quarante}

6 La déclaration de repentir trouve un antécédent dans la déclaration que les prisonniers politiques devaient faire pour obtenir une réduction de leur peine dès le début des années vingt. Par la suite, la loi de l'Idionymo qui date de 1929 et qui condamne ceux qui « chercheraient à appliquer des idées ayant comme but attesté le renversement violent du régime en place ou le détachement d'une partie du territoire national » ou encore ceux qui « lutteraient pour l'expansion ou l'application des idées subversives » est la première loi conçue spécialement pour faire face à la propagation des idées communistes au sein de la société grecque. Elle correspond moins à une réalité liée à l'importance numérique des effectifs des communistes grecs qu'à une peur fantasmée ${ }^{1}$ de la montée en puissance des forces de contestation. Elle est révélatrice de l'embarras des partis traditionnels devant l'avènement d'une nouvelle formation capable de tirer profit du mécontentement des classes laborieuses.

7 Dans le contexte de l'impasse politique de deux décennies marquées par la Division

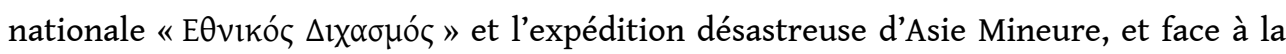
fragilisation des institutions du pays et la multiplication des coups d'État, les idées communistes ont pu paraître comme une alternative plausible et donc un danger potentiel pour les deux formations traditionnelles, celle des Libéraux, essentiellement constituée de républicains et celle des Populistes nettement promonarchiques ${ }^{2}$. Il en a résulté un anticommunisme largement partagé par les principales formations politiques du pays, d'autant plus marqué que la révolution bolchévique était géographiquement et historiquement proche.

De son côté, le coup d'État du 4 août 1936 inaugure une dictature de quatre ans que Métaxas définit dans son journal comme un régime par excellence " anticommuniste et antiparlementaire $»^{3}$. Métaxas pense utiliser la déception et la méfiance des masses populaires à l'égard des partis traditionnels, pour neutraliser l'ancienne division entre royalistes et venizélistes et lui substituer une nouvelle démarcation entre «les vrais Grecs » nationalistes et les communistes dont il théorisera le bannissement. 
9 Sur le plan législatif, une série de lois vient exclure les communistes du paysage politique du pays et faciliter leur mise en détention pour des motifs liés plus à une intention de propager des idées communistes qu'à des actes réellement subversifs. La loi d'urgence 117 promulguée le 18 septembre 1936 s'inscrit dans la continuité de l'Idionymo et vise avant tout ceux qui "propageraient, développeraient ou appliqueraient des théories, des idées ou des systèmes sociaux impliquant le renversement du régime en place » avec une mention spéciale pour les détenteurs de livres interdits par la censure. Ceux qui possédaient des ouvrages interdits encouraient d'importantes peines de déportation.

L'orientation principale des textes de loi vise à refuser aux prisonniers politiques la reconnaissance de leur statut et à institutionnaliser l'usage de la déclaration de repentir comme unique alternative à des peines d'emprisonnement et de déportation pouvant être continuellement reconduites sur la seule appréciation des instances judiciaires et/ou administratives ${ }^{4}$. À partir de 1933, ceux qui avaient été déportés et/ou emprisonnés sous le coup de la loi de 1929, peuvent être libérés après avoir purgé un quart de leur peine à condition bien entendu de signer la déclaration de repentir. Un nouveau décret législatif, la loi d'urgence 1075 vient compléter en février 1938 la législation anticommuniste en apportant trois mesures supplémentaires; l'usage systématique de la déclaration de repentir dans la procédure de remise de peine, la création de prisons et des camps disciplinaires spécialement conçus pour les prisonniers politiques et l'instauration d'un certificat de "pensée loyale», indispensable pour intégrer la fonction publique. La déclaration de repentir se trouve alors au centre d'un dispositif destiné à la conversion idéologique des prisonniers politiques dans la logique d'une série de lois qui, comme l'explique Alivizatos ${ }^{5}$, punissent des intentions ou des idées, plus que des actes. Il est d'ailleurs révélateur que les condamnations à mort puissent être commuées en des peines de perpétuité à condition que l'accusé accepte de signer la déclaration de repentir ${ }^{6}$ et qu'une collaboration active permet d'amnistier et de libérer le détenu.

11 Si le refus de la reconnaissance du statut de prisonnier politique et l'anticommunisme d'État constituent une constante dans l'histoire récente du pays, la déclaration de repentir, en tant qu'arme idéologique visant au déni identitaire de l'adversaire, prend toute son importance pendant et après la guerre civile grecque, juste après la Seconde Guerre mondiale.

\section{Le contexte historique : mouvements de résistance et prémices de la guerre civile}

12 Pour comprendre le rôle de la déclaration de repentir dans les années quarante, il est indispensable d'avoir une idée précise de la situation politique, militaire et juridique du pays pendant l'occupation et la guerre civile.

13 Pendant l'occupation, les différentes organisations d'inspiration nationaliste à l'exception de l'EDES de Zervas ${ }^{7}$ périclitent et l'EAM devient la plus importante organisation de la résistance grecque, celle qui rassemble toutes les forces de Gauche et même une partie du centre modéré. À la libération, l'EAM jouit du soutien incontestable de la majorité des Grecs et se présente comme une force de gouvernement aux objectifs économiques et sociaux précis. Le bilan de son action politique et militaire et l'étendue de son travail sur le terrain lui apportent la confiance 
d'une très grande partie de la population qui se sent partie prenante du processus, souhaite la démocratisation du pays et demande l'organisation d'un référendum pour en finir avec la monarchie.

14 L'élan populaire que l'EAM a su susciter pendant l'occupation s'accommode mal de la répartition du monde en zones d'influence décidée en haut lieu par Churchill et Staline et le retour imposé du roi Georges II, après les accords de ce dernier avec les Britanniques. L'impasse politique conduit à la séparation du pays légal et du pays réel et à la conflagration de la guerre civile. Les dirigeants du Parti communiste grec mal aiguillés par $\mathrm{Moscou}^{8}$ adoptent une stratégie hésitante, commettent des erreurs majeures en réfutant le jeu électoral et après la signature des accords de Varkiza perdent le contrôle de la situation se laissant entraîner dans l'illégalité. La Terreur Blanche s'abat sur tout le pays, les corps de sûreté de l'État et les bataillons de sécurité préalablement formés par les nazis, sévissent dans les campagnes et jusque dans les prisons.

15 La participation aux organisations de résistance EAM, ELAS - sa branche armée - ou EPON - ses jeunesses - et de manière plus générale toute action contre l'occupant allemand, deviennent à la fin de la guerre civile un chef d'inculpation majeur. Des milliers de résistants (communistes ou non) sont condamnés à mort, envoyés dans des camps de concentration et des prisons où tortures et humiliations sont utilisées méthodiquement dans le but de leur extorquer des aveux et/ou une reconversion idéologique, obtenue sous la forme d'une déclaration de repentir, par laquelle le détenu renie le communisme, son engagement et ses proches.

16 Il me semble intéressant de proposer une analyse du texte de la déclaration, de sa phraséologie et de ses soubassements idéologiques.

\section{L'idéologie et le discours des vainqueurs de la guerre civile dans la déclaration de repentir}

17 La parole des vainqueurs de la guerre civile prend la forme d'un discours stéréotypé, axé sur trois thématiques très idéologisées ; « la grandeur éternelle de la Race-Nation », «la guerre sainte menée depuis toujours par l'Orthodoxie contre les Infidèles » et « la lutte contre le fléau communiste », perçus à la fois comme « un péché capital» dont il faudrait se repentir et comme "un virus mortel ", dont il faudrait être guéri, d'où

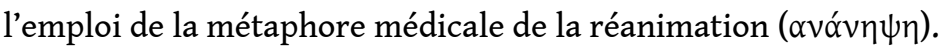

La déclaration de repentir se présente comme un texte que l'on demande de rédiger au détenu ou le plus souvent comme une lettre type, déjà préparée et prête à signer. Elle comporte diverses formules figées : «je, soussigné un tel, déclare, en toute liberté et sans avoir subi de contrainte morale ou physique abjurer le communisme et toutes ses ramifications" ou bien, "je reconnais avoir été victime des propos fallacieux des organisations communistes dont j'ignorais l'action antinationale; je déclare souhaiter la défaite des communistes, des bandits et autres traitres de la Nation » ou encore : «je me repentis de mon engagement antinational et demande à la mère Patrie de me pardonner mes offenses et de m'accepter de nouveau en son sein. Vive le Roi! Vive l'Armée nationale ! À bas le Communisme."

19 L'idéologie des vainqueurs de la guerre civile grecque se construit par opposition au discours communiste incarné par l'EAM, l'ELAS ou l'Armée démocratique. Il s'agit d'une 
réflexion dont le contenu idéologique n'a été théorisé qu'à partir de la guerre civile, la rhétorique sur « la loyauté nationale » étant une construction tardive", créée de toutes pièces pour contrer le discours communiste. En absence d'idéologie préalable, propre aux nationalistes, l'anticommunisme est pour ainsi dire l'élément fédérateur qui réunit autour de lui, les Venizélistes et les Royalistes, les Libéraux et les Populistes, les alliés des Allemands et ceux des Anglais et qui supprime toutes les divisions précédentes. L'idéologie des vainqueurs de la guerre civile se constitue donc sur le mode négatif avec l'anticommunisme d'État dans le rôle de dénominateur idéologique commun ${ }^{10}$.

La déclaration de repentir se met au service de la propagande des vainqueurs de la guerre civile pour étayer la thèse d'une manipulation de la masse des combattants/ résistants par la direction procommuniste (ou supposé telle) du mouvement de résistance EAM/ELAS et de son successeur, l'Armée démocratique. Elle infantilise la masse des combattants, introduit la notion de "pur Grec » abusé par les « ennemis de la Nation » et oppose, dans une série de signifiants très instructive, les « fils indignes de la Patrie » voire les " traîtres vendus aux Slaves ${ }^{11}$ aux vaillants descendants des héros de l'Antiquité ou de la guerre d'Indépendance de $1821^{12}$. Il y aurait donc un «bon peuple loyal», certes quelque peu naïf, qui aurait été berné par les promesses, forcément mensongères, d'une élite communiste composée de mauvais Grecs, voire même de non-Grecs, cette déchéance symbolique étant bien traduite par le vocable d'EAM-o-bulgares. Comme le fait remarquer Stratis Bournazos ${ }^{13}$, l'ennemi intérieur est systématiquement assimilé à un danger extérieur et l'on ne se réfère aux communistes que par des périphrases ou des mots composés, comportant des termes comme "slave " ou «bandit»: slavo-communistes, esclaves des Slaves, bandits de communistes ou encore mercenaires communistes et ainsi de suite. La propagande officielle s'obstine à parler d'incidents de banditisme conjoints à une agression étrangère même si, en réalité, il s'agit bien d'une guerre civile et si le conflit reste profondément grec.

Dans un discours où la moitié des Grecs deviennent d'un jour à l'autre des «traîtres bulgares ", la réalité d'une guerre fratricide est occultée et le conflit armé se travestit en lutte contre le banditisme. Nikos Sidéris ${ }^{14}$ dans son article sur la psychologie politique et la culture de la guerre civile, qualifie ce renversement du sens des signifiants de "meurtre du sens propre» ou mieux de "déni du sens figuré ». Et il spécifie que l'intériorisation psychologique de l'expérience de la persécution a été forgée par le refus d'un savoir, voire l'exclusion des référents symboliques indispensables au respect du sens propre et de la dimension métaphorique du langage, notamment à travers le déni des signifiants tels que «grec » ou «guerre civile». Ce paradoxe entraîne immanquablement la dépersonnalisation de l'adversaire, l'anéantissement de l'individu et l'annihilation du réel.

La phraséologie de la déclaration de repentir évoque la propagande habituelle du discours gouvernemental qui opère à la fois une réappropriation idéologique de l'héritage historique du pays (antique ou byzantin) et une falsification du passé récent des résistants. La pression morale et la torture physique pratiquées sur les détenus pour leur faire signer la déclaration de repentir, sont ainsi accompagnées de cours de « rééducation civique » où l'on cherche à déconstruire l'identité des détenus et à leur imposer un modèle factice du bon Grec, qui serait pétri des enseignements de la civilisation antique et de la religion orthodoxe $\mathrm{e}^{15}$. Les opposants à la monarchie et à la coalition anglo-américaine seront tour à tour privés de leur identité nationale, de leur statut de prisonniers politiques et de leur passé de résistants dans la mesure où la 
propagande officielle tient à ce que la guerre civile appelée «lutte contre le banditisme " résulte des agissements de groupes de criminels, assujettis aux Slaves et nie aux communistes grecs leur passé de partisans, voire tout simplement de bons patriotes.

Présenter la guerre civile grecque comme une guerre de plus contre des envahisseurs slaves permet non seulement de minimiser l'importance numérique des partisans de l'Armée démocratique, mais aussi d'inscrire le présent conflit armé dans le "glorieux passé du pays ». Le discours officiel établit de nombreux parallélismes avec les guerres menées dans le passé et projette ce conflit dans un continuum historique qui partirait de la Haute Antiquité pour arriver à l'épopée albanaise de 1941 en passant par la guerre d'indépendance de 1821. Les Slaves du X ${ }^{\mathrm{e}}$ siècle et ceux des années 1940 sont présentés comme les sempiternels ennemis du pays contre lesquels les Grecs se seraient toujours victorieusement battus.

L'identité de l'adversaire se construit dans le déni de la dimension idéologique du conflit. L'idéologie communiste de l'adversaire grec passe presque pour une conséquence naturelle d'une différence raciale et nationale. Dans le discours de la déclaration de repentir et de la presse progouvernementale, les Slaves sont considérés comme des êtres inférieurs, des peuples sauvages, qui ne pourraient qu'adhérer au communisme. Il s'agit d'un discours manichéen qui vient opposer les "Grecs civilisés » imprégnés de valeurs "nationales» aux ennemis de toujours, les «Slaves", tous les communistes grecs étant assimilés à des ennemis extérieurs. Par opposition à "ces ennemis étrangers ou à la solde des étrangers ", les vrais Grecs, seraient porteurs de valeurs supérieures et leurs combats aboutiraient forcément à une "nouvelle " victoire. Dans la phraséologie qui se dégage des déclarations de repentir, l'anticommunisme serait alors la lutte éternelle des civilisés contre les barbares ou encore des chrétiens contre les infidèles.

La déclaration de repentir emprunte au discours religieux les métaphores verbales et les pratiques de la repentance. La mortification de la chair préalable en quelque sorte à la purification de l'âme, tout comme l'idée d'une expiation qui passerait par le déni de l'altérité et le dénuement des signifiants de leurs sens résonnent comme autant de préalables à une rédemption totale du signataire et d'ailleurs s'accommodent assez bien du discours de l'église grecque. Il suffit de songer à la position de l'Église à propos du camp de rééducation de Makronissos. Ce lieu de déportation et de torture où des milliers de jeunes recrues et des civils ont été suppliciés a été comparé par l'église officielle au " réservoir de Siloé », lieu de salut spirituel pour des «brebis égarées ».

Même si l'Église grecque n'a pas eu de pouvoir comparable à celui de l'Église catholique pendant la guerre civile espagnole, force est de constater que ses dirigeants se sont eux aussi massivement placés du côté des forces royalistes. Et le ministère de la Justice a été le premier à reconnaitre l'importance du discours ecclésiastique, à la fois comme moyen de pression supplémentaire en vue de l'obtention d'une déclaration de repentir et comme caution morale face aux protestations de l'opinion publique et des organisations internationales ${ }^{16}$.

Certains prêtres abandonnaient le rôle plus ambivalent de conseiller spirituel ou de directeur de conscience pour endosser celui de Grand Inquisiteur ${ }^{17}$. Le cas de Stylianos Kornaros, archevêque en poste à Makronissos en est l'exemple type. Il dénonçait systématiquement les gens qui lui avaient donné des informations sous le secret de la confession et assistait aux séances de torture qui leur étaient infligés ${ }^{18}$, en brandissant 
sa Bible. Après les purges de l'Église grecque, l'adhésion au communisme est communément présentée comme un péché dont il faut se repentir. Le discours de l'Église, tout comme celui de la propagande gouvernementale cherche à faire peur et à déshumaniser l'adversaire idéologique. Stratis Bournazos démontre comment toute critique du socialisme réel est délaissée au profit d'une présentation fantasmatique ou irréaliste du " monstre communiste $»^{19}$. C'est un discours infantilisant qui substitue à la critique politique d'un système, une peur archaïque, je dirais aussi primitive que les réponses apportées par le « discours rééducatif. » La joute verbale y laisse bien la place à la volonté d'extermination physique de l'adversaire.

Le même discours de terreur qui rejette l'altérité au point de l'assimiler à une maladie se retrouve dans les métaphores médicales. Très présentes dans les déclarations de repentir, ces métaphores seront appelées à être réutilisées vingt ans plus tard par la dictature des colonels.

Dans le contexte de la guerre civile, les prisonniers doivent choisir entre le rejet de leur action politique et/ou militaire contre l'occupant nazi, les milices collaborationnistes et l'armée gouvernementale, et la persévérance dans leur "erreur idéologique ». S'ils refusent de se débarrasser, pour reprendre la terminologie médicale de l'époque, du « virus » ou de « la maladie mentale » du communisme et s'obstinent à ne pas signer de déclaration, ils sont exposés à subir tout le processus de rééducation, lequel vise encore et toujours, à diviser les prisonniers en deux catégories : ceux qui avoueront leurs fautes et pourront se racheter en mettant une croix sur leur identité antérieure, mais aussi en participant activement à la dénonciation ou à la rééducation de leurs anciens camarades et en faisant le jeu de la propagande gouvernementale et ceux qui refuseront de se repentir et qui se retrouveront bannis de la société, niés dans leur existence physique et morale.

La déclaration de repentir n'est qu'une première étape dans la reconversion idéologique, une concession qui sera appelée à être suivie par d'autres. Elle s'inscrit dans un mécanisme de déconstruction aussi bien de la personnalité des signataires que du lien social à l'intérieur de la communauté des détenus. Solution individuelle présupposant la rupture du lien avec la communauté des pairs, la signature de la déclaration aboutit à la double exclusion du signataire, tant par l'ensemble des détenus à qui le Parti communiste interdit de signer que par les pouvoirs publics qui demanderont au repenti de prouver constamment la véracité de sa reconversion en continuant à le traiter en citoyen de seconde zone.

\section{Le fonctionnement de la déclaration de repentir}

31 Pour mieux cerner le fonctionnement de la déclaration de repentir, il me semble important d'illustrer les conditions dans lesquelles elle est signée, puis d'évoquer ses conséquences sur le plan tant individuel que collectif.

\section{Dans quelles conditions la déclaration de repentir est-elle signée ?}

Pendant la guerre civile, la déclaration de repentir se transforme en une des principales raisons d'être des camps de rééducation et de déportation. Elle devient un enjeu d'autant plus important que le parti communiste continue à l'interdire quelles que soient les conditions de son obtention ${ }^{20}$ et que le gouvernement l'utilise constamment 
dans sa propagande comme une preuve de victoire morale sur le communisme. Tandis que le P.C. punit l'apostasie en " excommuniant» les signataires, la presse de droite et des personnalités du monde "libre " ne tarissent pas d'éloges à propos de «l'École Nationale de Makronissos $»^{21}$, premier camp de rééducation du pays. La rhétorique gouvernementale met en avant les résultats obtenus en matière de reconversion et affiche sa satisfaction de voir son modèle disciplinaire s'exporter en Malaisie ${ }^{22}$ tandis que les prisons et les camps de déportation atteignent des taux d'occupation qui dépassent largement leurs capacités d'accueil.

Pour obtenir la déclaration de repentir, les autorités ont recours à différents moyens de pression qui agissent aussi bien sur le prisonnier que sur son entourage : la persécution ou la manipulation des membres de la famille du détenu, l'organisation de séances de confrontation des détenus qui ont cédé sous la torture avec des prisonniers fraîchement arrivés dans le camp ${ }^{23}$ et qui souvent connaissent les signataires, ou encore des séances de propagande officielle où des détenus suppliciés déclarent avoir trouvé leur salut dans le discours rééducatif des représentants de l'ordre et incitent les autres prisonniers à suivre leur exemple en collaborant avec les autorités.

Ceux qui refusent de se repentir, subissent un « rituel expiatoire » qui est basé sur les châtiments corporels et les privations. Les non-signataires sont enfermés dans des cellules ou entre des barbelés spéciaux qui matérialisent leur mise en quarantaine, comme le cachot Arapis ${ }^{24}$ à la prison de Trikala ou encore le syrma à Makronissos. ${ }^{25}$ De manière générale, la signature de la déclaration de repentir conditionne l'accès aux soins, à l'eau, à la nourriture et même à la correspondance. Quand on enlève leurs enfants aux prisonnières ${ }^{26}$, quand on refuse d'opérer des femmes gravement malades ${ }^{27}$ et qu'on laisse mourir de faim et de soif des civils, on feint toujours de leur laisser en dernier lieu un autre choix, celui de survivre en abjurant le communisme.

Mais la déclaration est par excellence le choix impossible. Elle détruit moralement le prisonnier politique, en lui enlevant son identité, son visage social, un pan entier de son histoire et surtout sa dignité. Elle fait de lui un traître aux yeux du Parti et elle le rend inoffensif aux yeux de l'État.

\section{Conséquences de la déclaration de repentir}

Le fait d'avoir accepté de signer a de nombreuses conséquences, en termes d'image de soi et de représentations sociales.

La déclaration devient tour à tour une frontière réelle, imaginaire et symbolique entre les détenus. Elle oblige à une conversion de façade qui n'est pas intériorisée de la même façon par chaque détenu. Le vécu personnel antérieur à la détention, le sexe, l'âge, le statut dans la hiérarchie du parti, les circonstances de l'obtention de ladite déclaration, les autres concessions demandées - qui ne sont pas du même ordre dans les prisons, dans les camps de détention et dans les camps de rééducation - et la suite des événements dans la vie du prisonnier jouent un rôle majeur dans la perception de cet acte - tabou. Le discours et le jugement de valeur des autres univers sociaux dans lesquels le détenu évolue comme sujet ${ }^{28}$ sont aussi décisifs. La famille, les amis, le conjoint, les codétenus et les dirigeants du Parti représentent, non seulement, un cadre de référence pour le détenu, mais constituent également un contrepoids de taille face au travail de sape gouvernemental. Il ne faut pas oublier que le détenu investit son rôle 
dans la communauté de ses pairs, reconnaît - à des degrés divers - l'autorité de la direction du Parti et se voit à travers le regard de ses camarades de lutte.

En prison, la signature de la déclaration entraîne l'isolement du signataire, son exclusion des équipes de survie de la prison et in fine, son éloignement de l'aile réservée aux prisonniers politiques. La déclaration agit comme un facteur de division qui brise la cohésion et la solidarité collective. Elle sert aussi d'alibi à un discours communiste qui évite de reconnaître les erreurs stratégiques du Parti et attribue la défaite de la guerre civile aux « traîtres », aux " apostats », et autres individus indignes de confiance ${ }^{29}$. Dans une société grecque, qui au-delà de ses divisions, ne plaisante pas avec le code de l'honneur ${ }^{30}$, le mépris devient le lot des renégats qui ne trouvent grâce ni auprès de leurs anciens camarades ni auprès des nationalistes.

La déclaration de repentir vient déconstruire une image de soi, fondée sur sa propre expérience de vie et enlève sa légitimité au discours de référence du sujet. Elle oblige le signataire à rompre avec toute une série d'identifications et de liens qui lui avaient servi de repères dans son parcours, et qui avaient, en dernier lieu, donné un sens à sa vie et à son combat. Rejeter tous ses sacrifices, dénigrer ses parents morts pour la cause, ou bien condamner tout ce dont on a été fier, c'est se défaire d'une partie de son identité. En rédigeant le texte de la déclaration, le signataire est en plus, contraint d'adopter le discours des vainqueurs jusqu'aux moindres détails de sa rhétorique et c'est encore une fois, une violence symbolique insoutenable que de reproduire le discours de son agresseur ${ }^{31}$.

Dans certains cas, cette déconstruction va jusqu'à une transformation complète du signataire qui adopte l'idéologie de ses ennemis et participe activement à la reconversion de ses pairs. Signer est alors perçu comme le premier pas dans la trahison de ses camarades de lutte. À Makronissos, la déclaration de repentir instaure une frontière réelle entre les repentis et ceux qui ne le sont pas. On demande au détenu de prouver sa transformation en participant à la «rééducation des traîtres" et en acceptant de devenir à son tour un tortionnaire ${ }^{32}$. Le cas de Katsimihas ${ }^{33}$, ancien combattant de l'ELAS à Thèbes devenu principal rééducateur de Makronissos en dit long sur la portée de la capitulation demandée.

41 L'identification à l'agresseur, la reproduction de la violence subie, le besoin de voir d'autres personnes suivre et confirmer son exemple, pourraient expliquer le zèle de certains soldats réformés. L'intransigeance du Parti communiste face aux signataires, le rejet dont il accable les "traitres ", en niant le caractère contraint et forcé de leur démarche, aurait peut-être aussi contribué à radicaliser certaines réactions. En réalité, l'explication première de la transformation d'anciens sympathisants, militants ou combattants en tortionnaires acharnés réside dans la peur des représailles ${ }^{34}$.

42 Cela dit, se résigner à signer n'implique pas toujours un changement aussi radical et certains témoignages parlent - malgré le caractère transgressif d'un discours peu approuvé par le Parti - des signataires qui ont continué à aider leurs camarades non repentis, en dépit de multiples risques inhérents à cette démarche ${ }^{35}$. De nombreux témoignages nous citent le cas des prisonniers torturés qui momentanément cèdent, se disent prêts à signer pour se raviser quelques instants plus $\operatorname{tard}^{36}$ ou bien ils se réfèrent aux révocations de déclarations, par lesquelles des prisonniers reviennent sur leur abjuration, et subissent de lourdes sanctions ${ }^{37}$ dans l'espoir de se sentir «lavés » de la souillure symbolique de la déclaration. 

réalité, de nombreux signataires de la déclaration de repentir avaient continué à aider le Parti communiste et s'étaient montrés très solidaires des prisonniers politiques. La supériorité arithmétique des non-signataires parmi les auteurs des témoignages est à ce titre révélatrice de ce que j'appelle « le silence des signataires ». Leur discours plus ou moins autocensuré ne transparaît que dans les témoignages de ceux qui n'avaient pas signé et tout se passe, en fait, comme si les signataires de la déclaration avaient encore et toujours le sentiment de ne pas être autorisés à livrer leur propre témoignage. La déclaration de repentir et les représentations qu'elle évoquait, entre apostasie et trahison, ont agi sur les signataires comme une injonction de silence et comme une formule exécutoire. Des signifiants au sens figé se sont substitués à la parole libre du sujet et lui ont enlevé la possibilité de s'exprimer, pratiquement jusqu'aujourd'hui. Les anciens signataires ressentent de la honte à évoquer ce qu'ils assimilent à un viol de conscience. Et c'est peut-être ce qu'il y a de plus perfide dans l'humiliation et la culpabilité qu'entraîne la signature de la déclaration de repentir : penser qu'on y est toujours pour quelque chose, que c'est forcément un peu de sa faute et donc se refuser le statut de victime. Il est très difficile de se dire qu'on n'est ni traître, ni apostat, qu'on est juste un être humain qui a dû négocier sa survie physique en sacrifiant un peu de son idéal moral. 


\section{NOTES}

1. La confédération générale des travailleurs grecs voit le jour en 1918 en même temps que le Parti socialiste travailliste de la Grèce. "En Grèce, le Parti socialiste ouvrier de Grèce (SEKE) est fondé le 23 novembre 1918. Rassemblement de petits groupes socialistes provenant d'Athènes, de Salonique et de Volos, il décide d'adhérer à l'Internationale communiste à l'occasion de son $\mathrm{II}^{\mathrm{e}}$ congrès, le 5 avril 1920 et devient alors parti socialiste ouvrier de Grèce - communiste - dont la plupart des membres sont des réfugiés d'Asie Mineure. L'aile socialiste minoritaire décide de se retirer et de créer en septembre 1923, une formation aussi groupusculaire que sa compagne communiste. Les effectifs de la formation communiste grecque, qui devient officiellement le Parti communiste de Grèce en décembre 1924, sont en effet extrêmement réduits. Selon les calculs de Christophe Chiclet, ils atteignent 1000 membres en 1918, 2500 en 1926, 2000 en 1928 et 1500 en 1929. " L'Europe des communistes de José Gotovitch, Pascal Delwit et Jean-Michel de Waele. Questions du XX $\mathrm{X}^{\mathrm{e}}$ siècle, Éditions Complexes. Selon les calculs de Aggelos Elefantis, le PC grec avait 1.500 membres en 1930 et 17.500 en 1936. (cf Aggelos Elefantis, La promesse de la révolution impossible. Athènes, 1979, p. 289).

2. Vassilis Néféloudis explique dans son ouvrage "Rayon IX" l'étendue du pouvoir réel des communistes (assez limitée), mais aussi leur «capital sympathie» (bien plus vaste) auprès des classes populaires. Le PC grec était à l'époque minoritaire malgré ses grandes déclarations sur l'avènement imminent d'une Grèce socialiste. Or, pour Métaxas, propulsé Premier ministre par le palais avant de devenir dictateur, secouer le spectre du communisme s'était avéré être une

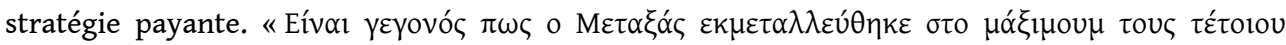

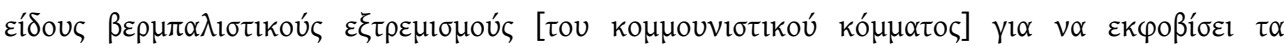

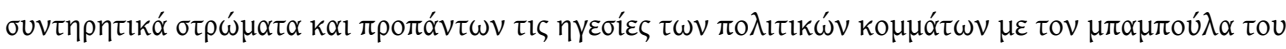

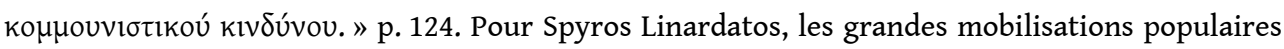
de mai 1936 sévèrement réprimées par le pouvoir en place, démontrent la présence de forces de contestation capables de s'opposer à la dictature du quatre août, mais ne justifient en aucun cas «le danger communiste imminent » évoqué par le dictateur. Métaxas lui-même, reconnaît selon Linardatos, «quelques semaines après son coup d'État que les propos concernant une prise du pouvoir par les communistes étaient pour le moins excessifs » p. 25 in Le quatre août, éditions Thémélio, Athènes, 1988.

3. Maniadakis, son ministre de l'Intérieur entretient une correspondance suivie avec Himler, chef alors de la Gestapo et lui demande des conseils sur «la lutte anticommuniste » à suivre. Il souhaite notamment se renseigner sur «la législation anticommuniste, les poursuites pénales prévues, les mesures policières contre les communistes ainsi que le système de détention et d'isolement disciplinaires (en camp de concentration ou en camp de travail)». Document des

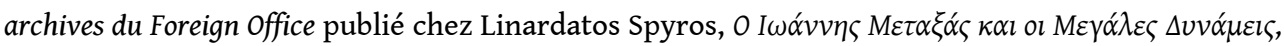
(1936-1940), (J. Métaxas et les grandes puissances 1936-1940), éd. Proskinio, Athènes, 1993 p. 69.

4. Les déportations étaient décidées par des instances administratives et ne devaient pas normalement constituer des peines d'emprisonnement même si dans la pratique la déportation aboutissait systématiquement (et de manière illégale) à l'enfermement dans des camps

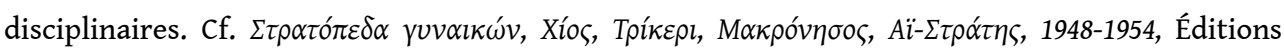

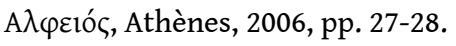

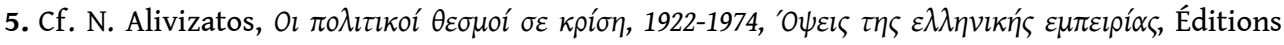

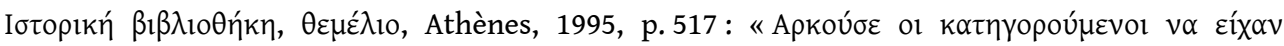

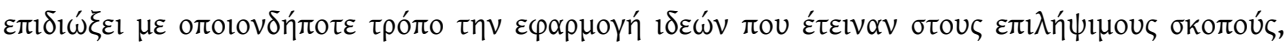

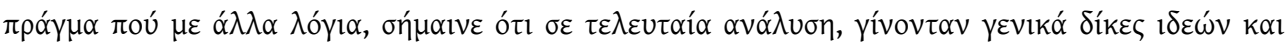

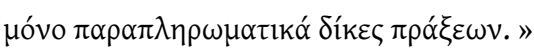




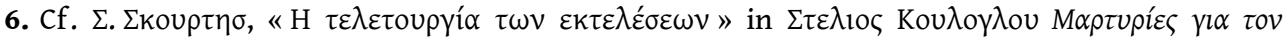

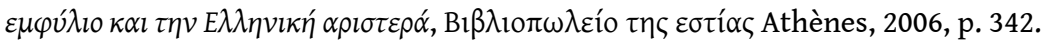

7. Pour se faire une idée du collaborationnisme des organisations nationalistes, du double jeu de Zervas et des conflits armés qui ont opposé les différents groupes de résistants $c f$. H. Fleischer,

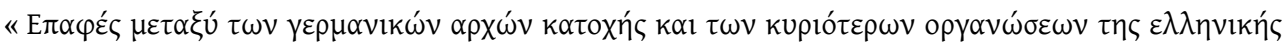

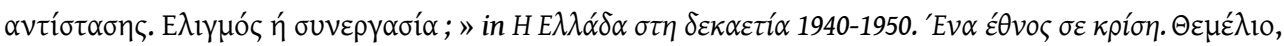
Athènes, 2006, pp. 91-115.

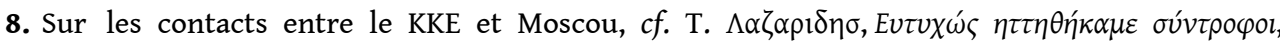

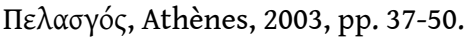

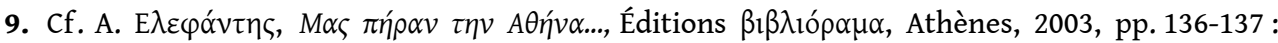

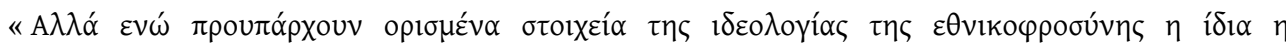

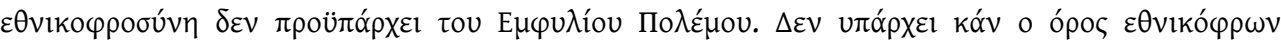

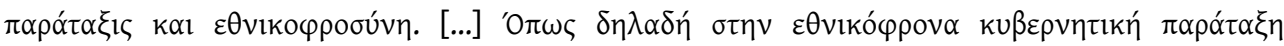

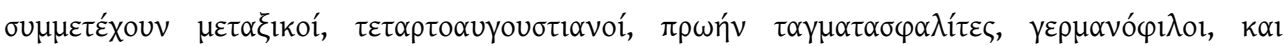

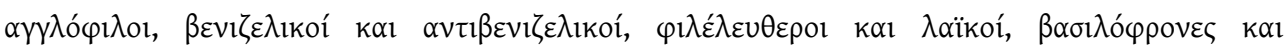

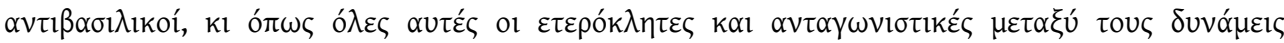

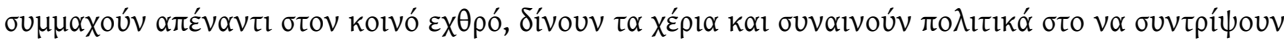

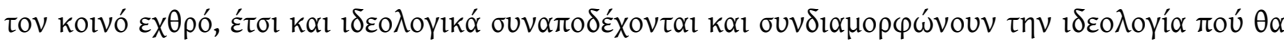

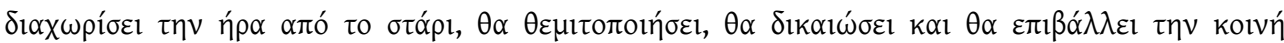
$\pi \rho \alpha \kappa \tau$ пй. »

10. Les classes dominantes peinent à articuler un discours cohérent et à se positionner sur le plan

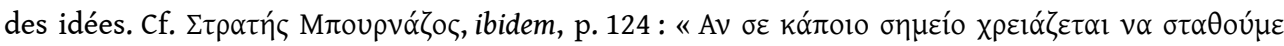

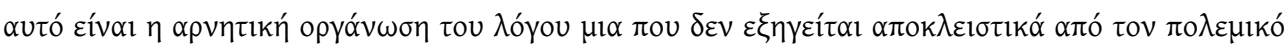

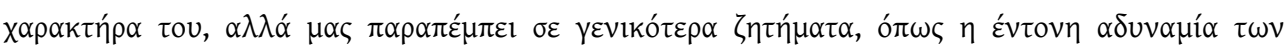

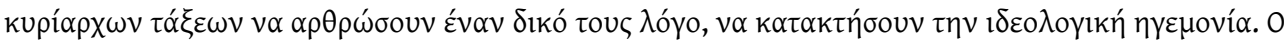

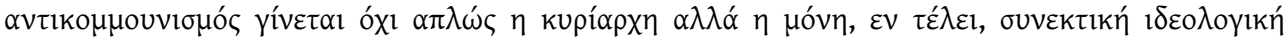
$\alpha \xi \dot{q} \alpha . »$

11. Le gouvernement grec dénonce à l'ONU le 3 décembre 1946 une agression étrangère ; cf. J. Dalègre, La Grèce depuis 1940, L'Harmattan, Paris, 2005, p. 79.

12. Les références aux héros de la guerre d'indépendance et aux figures des grands klephtes de la période ottomane faisaient partie d'un imaginaire populaire très partagé. Le discours libérateur de l'EAM s'en était également servi. Pour une présentation détaillée du mythe du klephte et de l'ambivalence de ses rapports avec le pouvoir et la rébellion, cf. Riki Van Boeschoten, , From Armatolic to People's Rule. Investigations into the Collective Memory of Rural Greece, 1750-1949, Editions

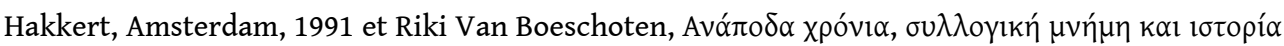

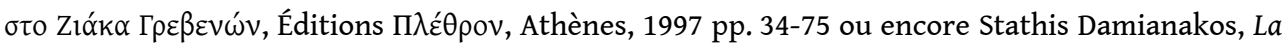
Grèce dissidente moderne, cultures rebelles, L'Harmattan, Paris, 2003.

13. Cf. Stratis Bournazos, « $O \alpha v \alpha \mu o \rho \varphi \omega \tau \imath \kappa o ́ \varsigma ~ \rho o ́ \lambda o \varsigma ~ \tau \omega v ~ v ı k \eta \tau \omega ́ v ~ \sigma \tau \eta v ~ M \alpha \kappa \rho o ́ v \eta \sigma o ~ » ~ i n ~ T o ~ E \mu \varphi v ́ \lambda_{\imath o}$

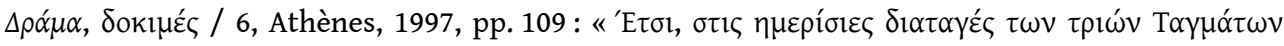

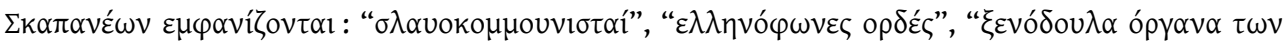

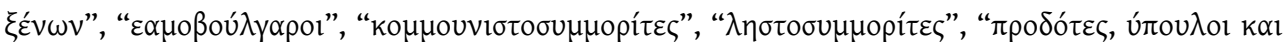

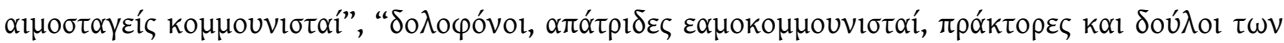

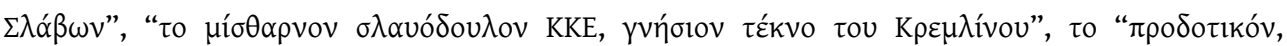

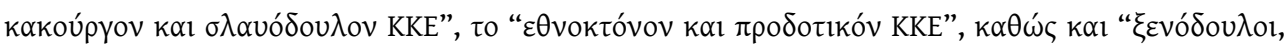

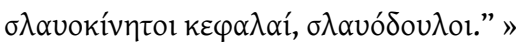

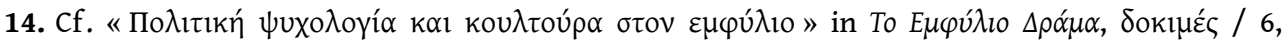

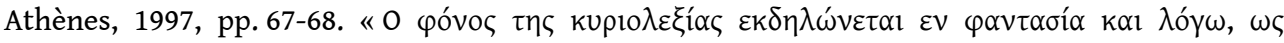

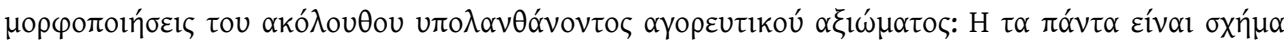

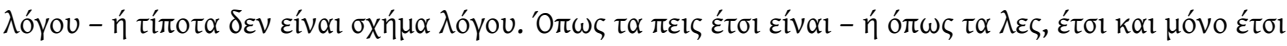




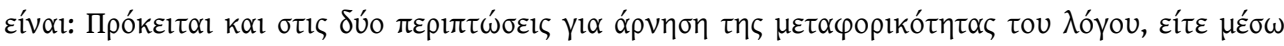

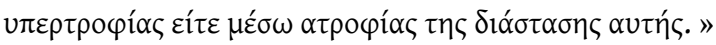

15. La propagande officielle ne craint pas le ridicule et l'on demande aux détenus de construire des répliques du Parthénon et autres monuments historiques à fort coefficient national, de vénérer le roi et la reine des Grecs, dans des mises en scène dont personne n'est dupe, et de participer activement au salut de leur âme et à celui de leurs camarades en se transformant en tortionnaires.

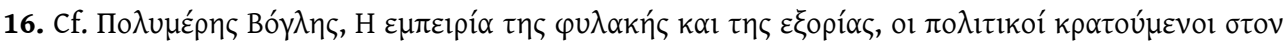

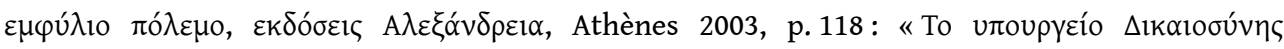

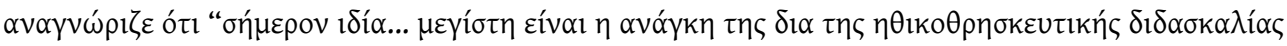

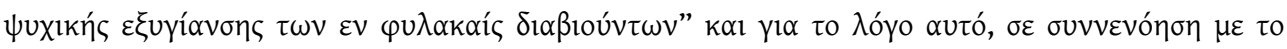

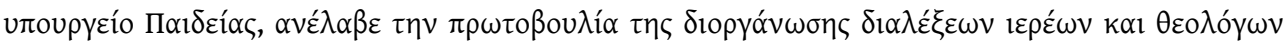

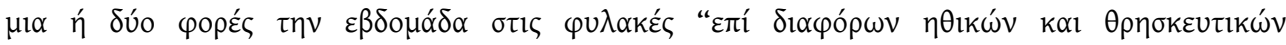

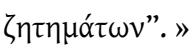

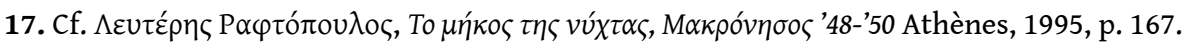

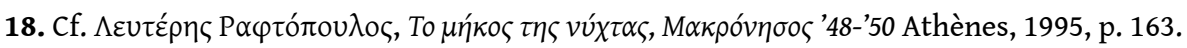

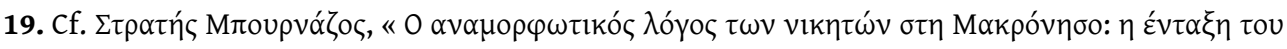

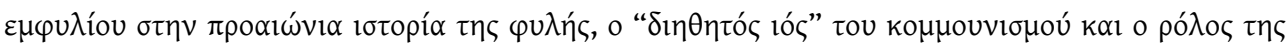

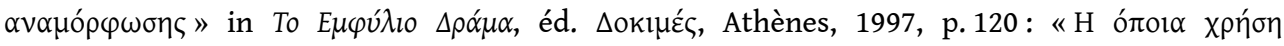

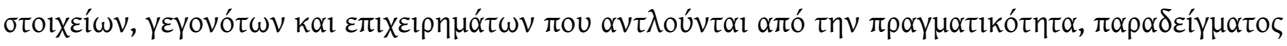

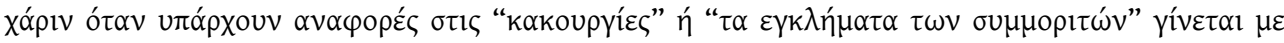

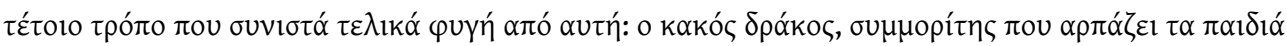

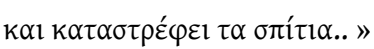

20. Sauf quand c'est sur ordre explicite du Parti pour sortir de prison et continuer la lutte armée ou bien travailler dans la clandestinité. Il s'agit de la $3^{\mathrm{e}}$ session plénière du Parti (septembre 1947)

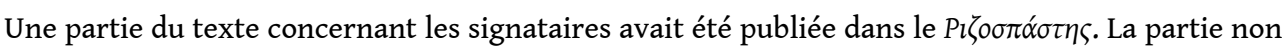
publiée du texte a été dévoilée par P. Iliou dans un dossier sur « La guerre civile en Grèce » (Avүท́, le 5 janvier 1980).

21. Cf. M $\pi 0 u \rho v \alpha \zeta o \sigma, 2000$, pp. 115-145.

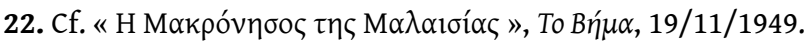

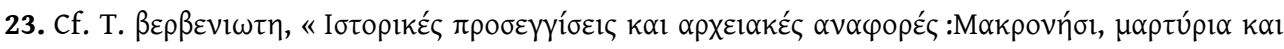

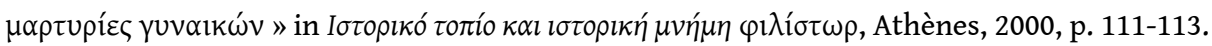

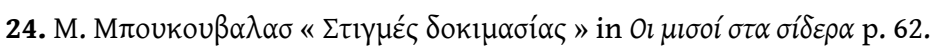

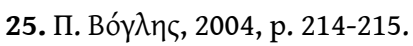

26. Ibidem p. 167 pour les femmes à Makronissos. À signaler également le cas de Marika épouse de Bartziotas qui refuse de signer sous la torture et finit par céder quand on la menace de lui

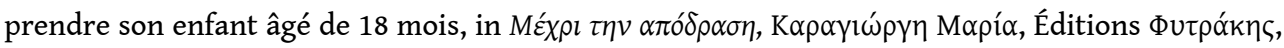
Athènes, 1989, pp. 161-162. Le cas d'enfants d'abord envoyés dans des camps de concentration ou des prisons avec leurs mères, pour en être séparés quelque temps après est magistralement traité dans le livre de Mando Dalianis-Karambatzakis, Children in Turmoil during the Greek Civil War 1946-49: Today's Adults, Karolinska Institutet, Stockholm, 1994.

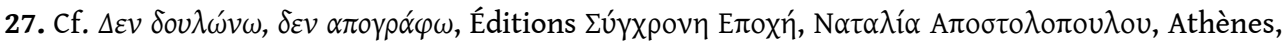
1985. p. 85.

28. Sur la subjectivité cf. $\Delta . \Lambda \alpha \mu \pi \rho \pi_{0}$.

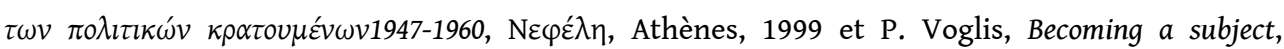
Berghahn Books, New York, 2002.

29. La condamnation des signataires de la déclaration de repentir par le PC est en partie liée aux conséquences de la déclaration de repentir sous la dictature de Métaxas quand la police de Maniadakis avait fini par démanteler le mécanisme du Parti et même créé un faux P.C. aux ordres 


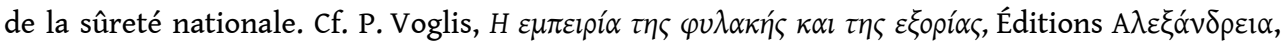
Athènes, 2002, p. 122.

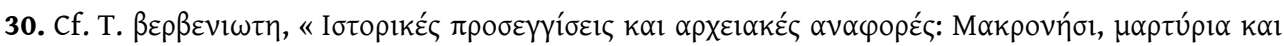

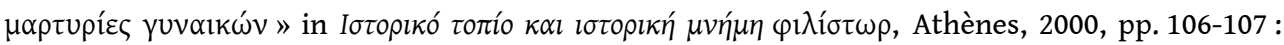
L'auteur prend comme hypothèse de travail la présence d'un idéal d'honneur qui rattache l'individu à la collectivité, très fort dans le monde rural en général et donc dans la société

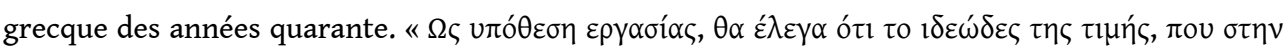

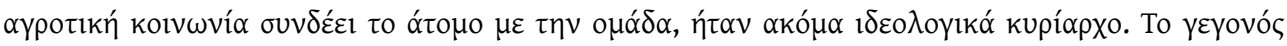

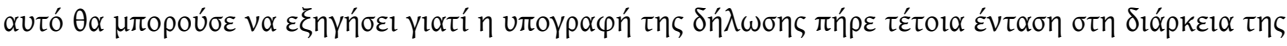

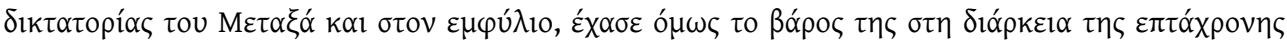

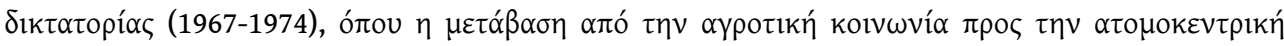

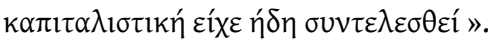

31. D'ailleurs le très beau titre du livre de Natalia Apostolopoulou, montre comment sonnait ce discours "rééducatif » pour les prisonniers. "Je ne déclare pas, je ne signe pas » s'est ainsi

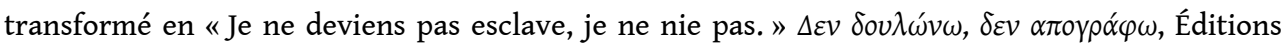

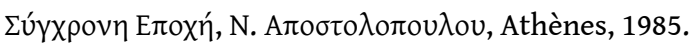

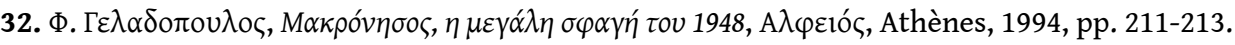

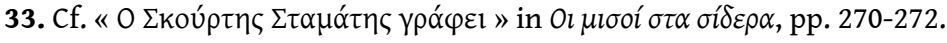

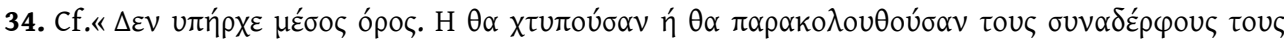

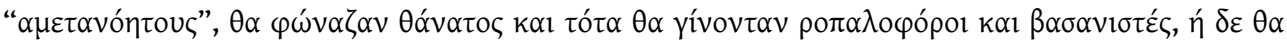

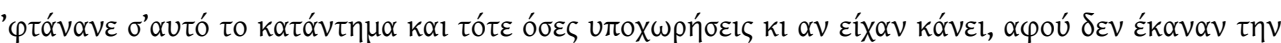

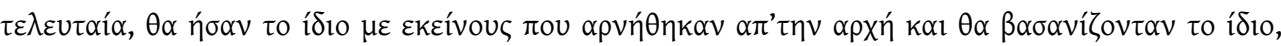

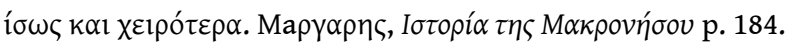

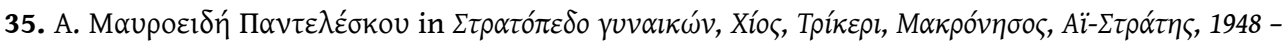

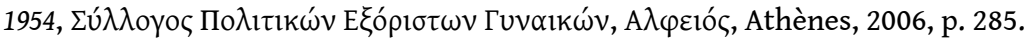

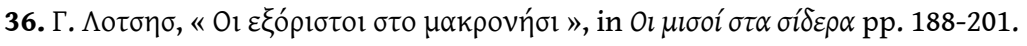

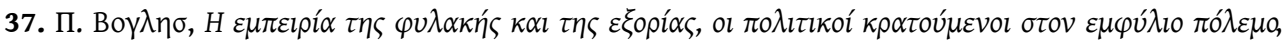

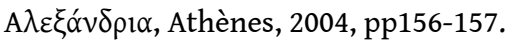

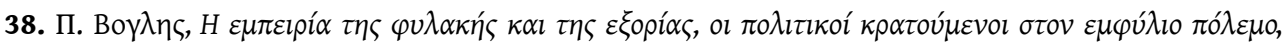

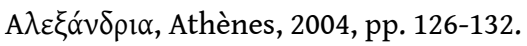

39. Ibidem, p. 129. À titre, d'exemple, Voglis cite l'exécution de Diamantis dans le journal Akropolis du 30 octobre 1947 et la publication de Daily Mirror, 10/11/1947, où des hommes armés portent les têtes coupées de Tzavelas et d'Aris.

\section{RÉSUMÉS}

La déclaration de repentir ou la première arme idéologique anticommuniste des gouvernements grecs qui détruisait moralement à jamais le suspect et sa famille.

L'article situe d'abord le contexte historique de la déclaration de repentir, arme de propagande et expression de l'anticommunisme, largement employée par les régimes grecs avant, pendant et après la guerre civile. Il étudie le discours de la déclaration, ses soubassements idéologiques, son fonctionnement, son impact sur la subjectivité des signataires ainsi que son incidence sur la vie politique et sociale. Il analyse les textes de loi successifs qui refusent aux prisonniers politiques la reconnaissance de leur statut et institutionnalisent la déclaration de repentir comme seule 
alternative à l'emprisonnement et à la déportation. Les prisonniers doivent choisir entre la persévérance dans leur "erreur idéologique » et le rejet de leur action politique ou militaire contre l'occupant nazi, les milices collaborationnistes et l'armée gouvernementale. Il analyse comment la déclaration détruit moralement le prisonnier, lui enlève son identité, son visage social, un pan entier de son histoire et surtout sa dignité, et comment elle peut entraîner une transformation complète du signataire, contraint d'adopter l'idéologie de ses ennemis. Enfin, la propagande gouvernementale sur ces déclarations scelle l'exclusion de la gauche du paysage politique du pays.

The article first situates the historical context of the declaration of repentance, weapon of propaganda and expression of anti-communism, largely employed by the Greek regimes before, during and after the Greek civil war. It studies the discourse of the declaration, its ideological foundations, its functioning, its impact on the subjectivity of the signatories as well as its impact on political and social life. It analyses the successive legal texts that denied political prisoners the recognition of their status and institutionalised the declaration of repentance as the only alternative to imprisonment and deportation. In the context of the civil war, the prisoners had to choose between persisting in their 'ideological error' and the rejection of their political or military action against the Nazi occupation, the collaborative militias and the governmental army. It analyses how the declaration destroyed the prisoner morally, robbed him of his identity, his social face, an entire part of his history and especially his dignity, and how it could lead to a complete transfomation of the signatory, forced to adopt the ideology of his enemies. Finally governmental propaganda on these declarations seals the exclusion of the left from the political landscape of the country.

\section{INDEX}

motsclesmk ГРцИјА

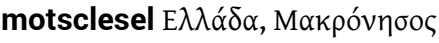

Thèmes : Histoire

Index chronologique : guerre civile grecque (1946-1949)

Mots-clés : Armée démocratique, anticommunisme, EAM, déportation, EDES, lutte idéologique, prisonniers politiques, ELAS, EPON, répression politique, Ethnikos dichasmos, Expédition d'Asie Mineure (1921-23), Idionymo, Makronissos, Metaxás Ioannis (1871-1941)

Index géographique : Grèce, Makronissos

motsclestr Makronisos, Yunan İç Savaşı (1946-1949), Yunanistan

Keywords : Anticommunism, Declaration of repentance, Deportation, Greece, Ideological war, Makronissos, Political repression, History, Reeducation policy

\section{AUTEUR}

\section{CHRISTINA ALEXOPOULOS}

CC INALCO, Doctorante CREE-CEB EA 4513 